\title{
The Absence of Serotonin in the Brain Alters Acute Stress Responsiveness by Interfering With the Genomic Function of the Glucocorticoid Receptors
}

\author{
Giulia Sbrini ${ }^{1}$, Paola Brivio ${ }^{1}$, Polina Mineva Peeva ${ }^{2}$, Mihail Todiras ${ }^{2}$, Michael Bader ${ }^{2,3}$, \\ Natalia Alenina $^{2,4 *}$ and Francesca Calabrese ${ }^{1 *}$ \\ ${ }^{1}$ Department of Pharmacological and Biomolecular Sciences, Università Degli Studi di Milano, Milan, Italy, ${ }^{2}$ Cardiovascular \\ and Metabolic Diseases, Max-Delbrück-Center for Molecular Medicine (MDC), Berlin, Germany, ${ }^{3}$ Charite-University Medicine, \\ Berlin, Germany, ${ }^{4}$ Institute of Translational Biomedicine, St. Petersburg State University, St. Petersburg, Russia
}

OPEN ACCESS

Edited by: Filippo Tempia,

University of Turin, Italy

Reviewed by:

Xiaolong Jiang,

Baylor College of Medicine,

United States

Grzegorz Hess,

Jagiellonian University, Poland

*Correspondence:

Francesca Calabrese

francesca.calabrese@unimi.it Natalia Alenina

alenina@mdc-berlin.de

Specialty section:

This article was submitted to Cellular Neurophysiology, a section of the journal Frontiers in Cellular Neuroscience

Received: 10 February 2020 Accepted: 17 April 2020 Published: 01 June 2020

Citation:

Sbrini G, Brivio P, Peeva PM, Todiras $M$, Bader $M$, Alenina N and Calabrese F (2020) The Absence of Serotonin in the Brain Alters Acute

Stress Responsiveness by Interfering With the Genomic Function of the

Glucocorticoid Receptors.

Front. Cell. Neurosci. 14:128. doi: 10.3389/fncel.2020.00128
Alterations in serotonergic transmission have been related to a major predisposition to develop psychiatric pathologies, such as depression. We took advantage of tryptophan hydroxylase (TPH) 2 deficient rats, characterized by a complete absence of serotonin in the brain, to evaluate whether a vulnerable genotype may influence the reaction to an acute stressor. In this context, we investigated if the glucocorticoid receptor (GR) genomic pathway activation was altered by the lack of serotonin in the central nervous system. Moreover, we analyzed the transcription pattern of the clock genes that can be affected by acute stressors. Adult wild type $\left(\mathrm{TPH}^{+/+}\right)$and $\mathrm{TPH} 2$-deficient $\left(\mathrm{TPH} 2^{-/-}\right)$ male rats were sacrificed after exposure to one single session of acute restraint stress. Protein and gene expression analyses were conducted in the prefrontal cortex (PFC). The acute stress enhanced the translocation of GRs in the nucleus of $\mathrm{TPH}^{+/+}$animals. This effect was blunted in TPH $2^{-/-}$rats, suggesting an impairment of the GR genomic mechanism. This alteration was mirrored in the expression of GR-responsive genes: acute stress led to the up-regulation of GR-target gene expression in $\mathrm{TPH} 2^{+/+}$, but not in $\mathrm{TPH} 2^{-/-}$animals. Finally, clock genes were differently modulated in the two genotypes after the acute restraint stress. Overall our findings suggest that the absence of serotonin within the brain interferes with the ability of the HPA axis to correctly modulate the response to acute stress, by altering the nuclear mechanisms of the GR and modulation of clock genes expression.

Keywords: serotonin, tryptophan hydroxylase, HPA axis, glucocorticoid, clock genes, rats, prefrontal cortex

\section{INTRODUCTION}

Psychiatric pathologies in humans have a multifactorial etiology with genetic and environmental factors that may interact thus inducing the manifestation of a wide range of symptoms (Akiskal and McKinney, 1973, 1975). Among the genetic factors that can predispose to the development of psychiatric illnesses, such as depression, alterations in the serotonergic system are the most 
studied not only at clinical level but also in preclinical studies (Caspi et al., 2003; Calabrese et al., 2013; Brivio et al., 2018; Ottenhof et al., 2018). Interestingly, serotonergic projections innervate the paraventricular nucleus of the hypothalamus as well as the brain regions involved in the stress-induced activity of the HPA axis, including the prefrontal cortex (PFC) (Pan and Gilbert, 1992; Van de Kar et al., 2001; Herman et al., 2003).

Serotonin synthesis starts from the amino acid tryptophan through the action of the rate-limiting enzyme tryptophan hydroxylase (TPH) (Fitzpatrick, 1999). This enzyme exists in two isoforms: TPH1 expressed at the periphery, and TPH2, expressed in the raphe nuclei in the brain and responsible for the production of serotonin at the central level (Walther et al., 2003). Accordingly, animal models with genetic deletion of TPH2 show reduced levels of serotonin in the brain (Mosienko et al., 2015).

As mentioned above, also environmental factors play a key role in the manifestation of mood disorders. Chronic stress is one of the main precipitating factors, that negatively affect behaviors (Breslau and Davis, 1986; Lupien et al., 2018). On the contrary, acute stressors can have a positive impact on the brain leading to the activation of neuroplastic mechanisms and the transcription of activity-dependent genes (Brivio et al., 2020). In this context, we recently demonstrated that despite a normal corticosterone-release pattern upon acute stress, $\mathrm{TPH} 2$-deficiency in rats results in an up-regulation of the neurotrophin BDNF in basal conditions, but a blunted response to acute stress (Brivio et al., 2018).

Here, we took advantage of the TPH2-deficient (TPH2 ${ }^{-/-}$) rats (Kaplan et al., 2016), characterized by a complete absence of serotonin in the brain, to evaluate the impact of central serotonin deficiency on the acute stress response. Hence, we evaluated if the molecular changes that physiologically occur after stress exposure could be altered by the absence of serotonin in the brain focusing on the hypothalamicpituitary-adrenal (HPA) axis, one of the main mechanisms that allow a proper response to stressful events (McEwen, 2007). In particular, we analyzed the genomic pathway activation that occurs after the translocation into the nucleus of the glucocorticoid receptors (GRs) (Trapp et al., 1994) and the subsequent transcription of genes carrying the glucocorticoid responsive element (GRE) in their regulatory DNA regions. Interestingly, genes involved in the control of circadian rhythms, period circadian regulator (Per1) and Per2, are among the glucocorticoid responsive genes, and their expression is known to be affected by acute stress exposure (Yamamoto et al., 2005) as well as by corticosterone levels in the blood (Nader et al., 2010). Therefore, we also analyzed the gene expression profile of the main players involved in the control of the clock gene machinery in $\mathrm{TPH} 2^{-/-}$and wild type $\left(\mathrm{TPH}^{+/+}\right)$ rats after the acute challenge. All molecular analyses were performed in the PFC, a brain region connected not only to serotonin functions but also deeply affected by stress exposure (Duman and Monteggia, 2006; Stuss and Knight, 2009; Brivio et al., 2020).

\section{MATERIALS AND METHODS}

\section{Animals}

Animals were kept at the animal facility of Max-Delbrück Center for Molecular Medicine under standardized conditions with an artificial 12/12-h light/dark cycle (lights on at 6 a.m.), room temperature of $22^{\circ} \mathrm{C}$, and approximately $80 \%$ humidity with access to food (Ssniff, Soest, Germany) and water ad libitum. All procedures were approved by the ethical committee of the local government (LAGeSo, Berlin, Germany).

Twelve to 14 weeks old male TPH2-deficient $\left(\mathrm{TPH}_{2}^{-/-}\right.$) (Kaplan et al., 2016) and wild type $\left(\mathrm{TPH}^{+/+}\right)$rats on the Dark Agouti background were used in the experiments. Animals heterozygous for the TPH2 mutation (10 bp deletion in exon 7) were bred to generate experimental groups of both genotypes. To genotype offspring, genomic DNA from ear biopsies was amplified with primers TPH2ZFN_FW $5^{\prime}$-CCC TTC TCC ACA GAA GTG CT and TPH2ZFN_REV 5'-GGC CTT TAG GTC CTG AGG TT and the resulting PCR fragment was digested with the restriction nuclease Mnl1, which cuts exclusively the PCR products of the WT allele.

\section{Stress Procedure}

Adult male $\mathrm{TPH}_{2}{ }^{++}$and $\mathrm{TPH} 2^{-/-}$rats were exposed to acute restraint stress as previously described (Brivio et al., 2018; Calabrese et al., 2020). Briefly, rats were placed in an air-accessible apparatus for $1 \mathrm{~h}$. The size of the container was similar to the size of the animal, making the rats almost immobile. Stressed animals ( $n=5$ per genotype) were sacrificed immediately at the end of the stress session. Unstressed animals $(n=13)$ served as a control group.

\section{Brain Tissue Collection}

Animals were sacrificed by decapitation and the PFC was rapidly dissected, frozen on dry ice and stored at $-80^{\circ} \mathrm{C}$ for the molecular analyses. Dissections were performed according to the atlas of Paxinos and Watson (Paxinos and Watson, 2007). PFC was dissected from 2-mm-thick slices [PFC defined as $\mathrm{Cg} 1, \mathrm{Cg} 3$ and IL sub-regions corresponding to plates 6-9 (approximate weight $8 \mathrm{mg}$ )]. The left hemisphere was taken for protein whereas the right was taken for RNA.

\section{RNA Preparation and Gene Expression Analysis by Quantitative Real-Time PCR}

Total RNA was isolated by a single step of guanidinium isothiocyanate/phenol extraction using PureZol RNA isolation reagent (Bio-Rad Laboratories, Italy) according to the manufacturer's instructions and quantified by spectrophotometric analysis.

The samples were then processed for real-time polymerase chain reaction (RT-PCR) to assess the expression of Growth Arrest And DNA Damage Inducible $\beta$ (Gadd45 $\beta$ ), Serum/Glucocorticoid Regulated Kinase (Sgk1), Nuclear Receptor Subfamily four Group A Member 1 (Nr4a1), Dual Specificity Phosphatase 1 (Dusp1), S100A10 (P11), Forkhead box O1 (FoxO1), FKBP Prolyl Isomerase 5 (Fkbp5), TSC22 Domain Family Member 3 (Gilz), Per1, Per2, cryptochrome circadian 
TABLE 1A | Sequences of forward and reverse primers and probes used in Real-time PCR analyses and purchased from Eurofins MWG-Operon.

\begin{tabular}{|c|c|c|c|}
\hline Gene & Forward primer & Reverse primer & Probe \\
\hline $36 b 4$ & TTCCCACTGGCTGAGGT & CGCAGCCGCTGC & AAGGCCTTCCTGGCCGATCCATC \\
\hline Sgk1 & GACTACATTAATGGCGGAGAGC & AGGGAGTGCAGATAACCCAAG & TGCTCGCTTCTACGCAGC \\
\hline Dusp1 & TGTGCCTGACAGTGCAGAAT & ATCTICCGGGAAGCATGGT & ATCCTGTCCTTCCTGTACCT \\
\hline P11 & AGAGTGCTCATGGAAAGGGA & AGCTCTGGAAGCCCACTIT & ATAATGAAAGACCTGGACCAGTGC \\
\hline FoxO1 & GAGTGGATGGTGAAGAGTGTG & GGACAGATTGTGGCGAATTG & TCAAGGATAAGGGCGACAGCAACAG \\
\hline Fkbp5 & GAACCCAATGCTGAGCTTATG & ATGTACTTGCCTCCCTTGAAG & TGTCCATCTCCCAGGATTCTITGGC \\
\hline Gilz & CGGTCTATCAACTGCACAATTC & CTTCACTAGATCCATGGCCTG & AACGGAAACCACATCCCCTCCAA \\
\hline Per1 & AGAGCTGAGTCCTTGCCATT & TGGCTGATGACACTGATGCA & AGCGGAGTTCTCACAGTTCA \\
\hline Per2 & TTGTGCCTCCCGATGATGAA & AGTGGGCAGCCTICGATTA & GTACATCACACTGGACACTAGC \\
\hline Cry1 & TCAATCCACGGAAAGCCTGT & CCACAAACAACCCACGCTIT & GGAACCCCATCTGTGTTCAA \\
\hline Cry2 & TAGTCCACGCCAATGATGCA & TGCCCAAACTGAAAGGCTTC & TCTATGAGCCCTGGAATGCT \\
\hline Rev-erb $\alpha$ & ACGTCCCCACACACTITACA & ACAAGTGGCCATGGAAGACA & GGCACCAGCAACATTACCAA \\
\hline Rev-erb $\beta$ & ACGGATGAGTGTाTCCTGCA & AGCGACGAGGAAATGAGCTT & TTCTGGTGTCTGCAGATCGA \\
\hline
\end{tabular}

TABLE 1B | Probes purchased from Life Technologies, which did not disclose the sequences.

\begin{tabular}{lll}
\hline Gene & Accession Number & Assay ID \\
\hline Gadd45 $\beta$ & BC085337.1 & Rn01452530_gl \\
Nr4a1 & BC097313.1 & Rn01533237_m1
\end{tabular}

regulator (Cry1), Cry2, nuclear receptor subfamily 1, group D member 1 (Rev-erb $\alpha)$, nuclear receptor subfamily, group D, member 2 (Rev-erb $\beta$ ) (primer and probes sequences are listed in Tables 1A,B). RNAs were treated with DNase (Thermoscientific, Italy) to avoid DNA contamination. Gene expression was analyzed by TaqMan qRT-PCR one-step RT-PCR kit for probes (Bio-Rad laboratories, Italy). Samples were run in 384 well formats in triplicate as a multiplexed reaction with a normalizing internal control (36b4).

Thermal cycling was initiated with an incubation at $50^{\circ} \mathrm{C}$ for $10 \mathrm{~min}$ (RNA retrotranscription) and then at $95^{\circ} \mathrm{C}$ for $5 \mathrm{~min}$ (TaqMan polymerase activation). After this initial step, 39 cycles of PCR were performed. Each PCR cycle consisted of heating the samples at $95^{\circ} \mathrm{C}$ for $10 \mathrm{~s}$ to enable the melting process and then for $30 \mathrm{~s}$ at $60^{\circ} \mathrm{C}$ for the annealing and extension reactions. A comparative cycle threshold $(\mathrm{Ct})$ method was used to calculate the relative target gene expression.

\section{Protein Extraction and Western Blot Analysis}

Western blot analysis was used to investigate GR, Myc-associated zinc finger protein 1 (MAZ1) and Specificity Protein 1 (SP1) protein levels in the nuclear fraction, in the cytosolic compartment and the whole homogenate. Tissues were manually homogenized using a glass-glass potter in a $\mathrm{pH} 7.4$ cold buffer containing $0.32 \mathrm{M}$ sucrose, $0.1 \mathrm{mM}$ EGTA, $1 \mathrm{mM}$ HEPES solution in the presence of a complete set of proteases (Roche) and phosphatase (Sigma-Aldrich) inhibitors. The total homogenate was centrifuged at $1,000 \mathrm{~g}$ for $10 \mathrm{~min}$ at $4^{\circ} \mathrm{C}$ to obtain a pellet enriched in nuclear components, which was suspended in a buffer [20 mM HEPES, $0.1 \mathrm{mM}$ dithiothreitol (DTT), $0.1 \mathrm{mM}$ EGTA] with protease and phosphatase inhibitors. The supernatant was further centrifuged at $10000 \mathrm{~g}$ for $10 \mathrm{~min}$ at $4^{\circ} \mathrm{C}$ to eliminate the membrane fraction and the resulting supernatant corresponding to the cytosolic fraction was conserved for the protein analyses. The purity of these fractions was previously reported (Brivio et al., 2019). Total protein content was measured according to the Bradford Protein Assay procedure (Bio-Rad Laboratories), using bovine serum albumin (BSA) as a calibration standard. Equal amounts of protein were run under reducing conditions on $10 \%$ SDS-polyacrylamide gels and then electrophoretically transferred onto Polyvinylidene Difluoride (PVDF) or nitrocellulose membranes (GE Healthcare Life Sciences). The blots for GR were blocked with BSA in TBS $+0.2 \%$ sodium azide, while the others (MAZ1, SP1 and $\beta$-actin) with $5 \%$ nonfat dry milk and then were incubated with the proper primary antibodies [GR: 1:500 (ThermoFisher), $4^{\circ}$ overnight $(\mathrm{O} / \mathrm{N})$; MAZ1: 1:500 BSA $5 \% 4^{\circ} \mathrm{C}$ O/N; SP1: 1:250 BSA $5 \% \quad 4^{\circ} \mathrm{C} \quad \mathrm{O} / \mathrm{N} \quad \beta$-actin: $1: 10,000$ (Sigma), room temperature (RT) $45 \mathrm{~min}]$. Membranes were then incubated with the appropriate secondary antibody (GR: anti-rabbit, 1:2,000, RT, $1 \mathrm{~h}$; MAZ1: anti-rabbit 1:1,000, RT $1 \mathrm{~h}$; SP1: anti-rabbit 1:1,000 RT $1 \mathrm{~h}$; $\beta$-actin: anti-mouse, 1:10,000, $45 \mathrm{~min}$ ). Immunocomplexes were visualized by chemiluminescence using the Western Lightning Clarity ECL (Bio-Rad Laboratories) and the Chemidoc MP imaging system (Bio-Rad Laboratories). GR levels were quantified by the evaluation of band densities, normalized to the $\beta$-actin (ImageLab, Bio-Rad Laboratories).

\section{Statistical Analysis}

All the analyses were conducted by using "IBM SPSS Statistics, version 24." Results were analyzed with the two-way ANOVA followed by Fisher's PLSD. Significance for all tests was assumed for $p<0.05$. Data are presented as means \pm standard error (SEM).

\section{RESULTS}

\section{Glucocorticoid Receptor Translocation Into the Nucleus Induced by Acute Stress Exposure Is Blunted in TPH2 ${ }^{-/-}$Rats}

The activation of the HPA axis after acute restraint stress results in the release of corticosterone from the adrenal glands and subsequent response in the extrinsic HPA axis structures, such as PFC via activation of the genomic pathway of the GRs 
(Adzic et al., 2009). In particular, the binding of the hormone to its receptor induces its translocation into the nucleus (Revollo and Cidlowski, 2009).

We first analyzed the GR protein levels in the nuclear and cytosolic fractions immediately after the end of the acute stress. As shown in Figure 1A, we found a significant genotype $\times$ stress interaction $\left(F_{(1,16)}=4.971 p<0.05\right.$; two-way ANOVA) in GR protein levels in the nuclear compartment, but no effects of the genotype $\left(F_{(1,16)}=3.055 p>0.05\right.$; two-way ANOVA $)$ and of the stress $\left(F_{(1,16)}=3.747 p>0.05\right.$; two-way ANOVA). Indeed, the receptor was significantly up-regulated by the stress in the nuclear compartment of $\mathrm{TPH} 2^{+/+}$rats $(+89 \%$ $p<0.05$ vs. $\mathrm{TPH}_{2}^{+/+} /$naïve; Fisher's PLSD), but not in the $\mathrm{TPH} 2^{-/-}$counterpart.

On the contrary, we did not find any significant modulation of GR protein levels in the whole homogenate and the cytosolic compartment even if a slight decrease of its levels was observed in the cytosol of $\mathrm{TPH} 2^{+/+}$stressed rats $(-27 \% p>0.05$ vs. $\mathrm{TPH} 2^{+/+} /$naïve; Fisher's PLSD) (Figures 1B,C).

Finally, we looked at the ratio of the nuclear vs. cytosolic GR levels as an indicator of the translocation into the nucleus of the receptor after stress exposure and we found a borderline significance for the genotype $\times$ stress interaction $\left(F_{(1,13)}=4.761\right.$ $p=0.054$; two-way ANOVA). Indeed, following the results

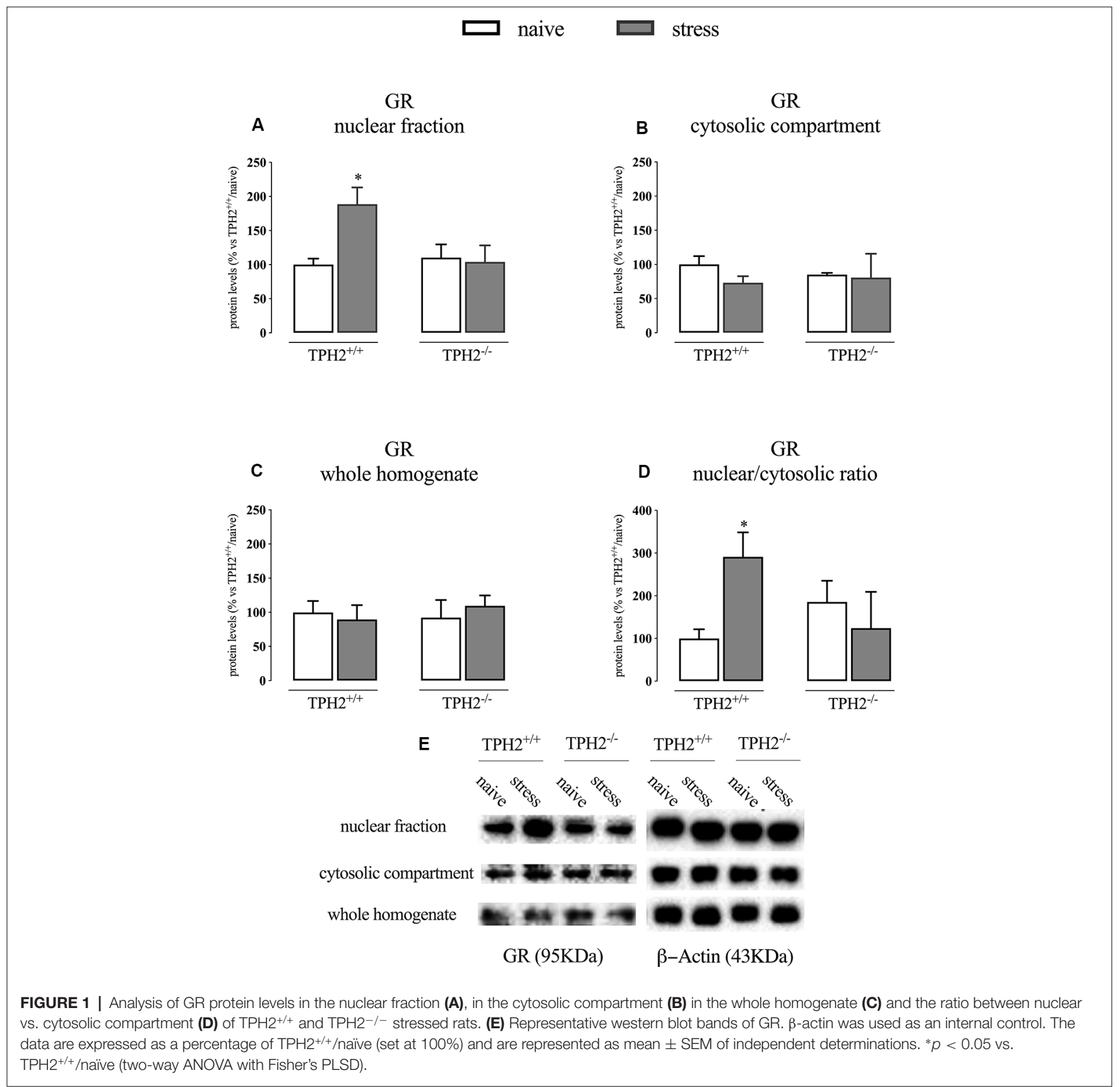


described in panels $\mathrm{A}$ and $\mathrm{B}$, we observed an increased translocation in $\mathrm{TPH}_{2}^{+/+}$stressed animals $(+191 \% p<0.05$ vs. $\mathrm{TPH}^{+/+} /$naïe) while this effect was blunted in $\mathrm{TPH} 2^{-/-}$ rats (Figure 1D). Figure $1 \mathrm{E}$ is a representative of western blot bands.

\section{MAZ1 Transcription Factor Is Increased After Stress Exposure in TPH2 ${ }^{+/+}$but Not in $\mathrm{TPH}^{-/-}$Stressed Rats}

Once in the nucleus, GRs bind the GREs, and this binding is facilitated by the presence of cofactors such as MAZ1 and SP1 (Datson et al., 2011).

In the whole homogenate, MAZ1 protein levels showed a significant genotype $\times$ stress interaction $\left(F_{(1,15)}=6.424 p<0.05\right.$; two-way ANOVA) but no effect of the genotype $\left(F_{(1,15)}=3.216\right.$ $p>0.05$; two-way ANOVA) and of the stress $\left(F_{(1,15)}=0.029\right.$ $p>0.05$; two-way ANOVA). Accordingly, we observed a significant increase of MAZ1 protein levels in $\mathrm{TPH}_{2}{ }^{+/+}$ rats exposed to stress when compared to $\mathrm{TPH} 2^{-/-}$stressed animals $\left(+121 \%, p<0.01\right.$ vs. TPH $2^{-/} /$stress; Fisher's PLSD) (Figure 2A). We did not find any changes in SP1 protein levels (Figure 2B). Figure 2C is a representative of western blot bands.

\section{Up-Regulation of Glucocorticoid Responsive Gene Expression Is Blunted in TPH2-/- Stressed Rats}

The translocation into the nucleus of the GRs leads to the expression of the glucocorticoid responsive genes, carrying the GREs.

As shown in Figure 3A, Dusp1 mRNA levels were significantly affected by the stress $\left(F_{(1,33)}=10.770 p<0.01\right.$, two-way ANOVA), that induced an increase in the expression of this gene specifically in $\mathrm{TPH} 2^{+/+}$rats $(+53 \%, p<0.01$ vs. $\mathrm{TPH} 2^{+/+} /$naïve).

Moreover, Gadd $45 \beta$ expression (Figure 3B) was significantly affected both by the genotype and by the stress (genotype: $F_{(1,34)}=8.765 p<0.05$ and stress: $\left.F_{(1,34)}=85.447 p<0.001\right)$. Indeed, stress exposure induced an increased transcription not only in $\mathrm{TPH}_{2}^{+/+}$rats $\left(+113 \% p<0.001 \mathrm{vs}\right.$. $\mathrm{TPH} 2^{+/+} /$naïve $)$but also in $\mathrm{TPH} 2^{-/-}$animals $\left(+40 \%, p<0.01\right.$ vs. TPH $2^{-/-} /$naïve).
However, as demonstrated by the significant genotype $\times$ stress interaction $\left(F_{(1,34)}=16.447 p<0.001\right)$, the up-regulation due to the challenge found in $\mathrm{TPH} 2^{+/+}$was more robust $(+39 \%$, $p<0.001$ vs. $\mathrm{TPH}_{2}+/ /$ stress $)$ compared to those found in $\mathrm{TPH} 2^{-/-}$stressed rats.

Similarly, Nr4a1 was affected by the genotype $\left(F_{(1,34)}=4.602\right.$ $p<0.05$, two-way ANOVA $)$ and by the stress $\left(F_{(1,34)}=101.764\right.$ $p<0.001$, two-way ANOVA) with a significant interaction between the two variables $\left(F_{(1,34)}=16.650 p<0.001\right.$, two-way ANOVA). Accordingly, Nr4a1 expression was up-regulated both in $\mathrm{TPH}_{2}{ }^{++}\left(+153 \%\right.$ vs. $\mathrm{TPH} 2^{+/+} /$naïve $)$and in $\mathrm{TPH} 2^{-/-}(+54 \%$ vs. $\mathrm{TPH} 2^{-/-} /$naïve) in comparison to their controls. Again, we observed a higher expression of $\mathrm{Nr} 4 a \mathrm{l}$ in $\mathrm{TPH} 2^{+/+}$in comparison to $\mathrm{TPH} 2^{-/-}$stressed rats $\left(+36 \% p<0.001\right.$ vs. $\mathrm{TPH}^{+/+} /$ stress) (Figure 3C).

On the contrary, Sgk1, Gilz and Fkbp5 mRNA levels were affected only by the stress $\left(S g k 1: F_{(1,35)}=135.512 p<0.001\right.$, two-way ANOVA; Gilz: $F_{(1,35)}=96.360 p<0.001$, two-way ANOVA; Fkbp5: $F_{(1,33)}=16.167 p<0.001$, two-way ANOVA). In particular, we observed a comparable increase of these genes expression after the acute challenge both in $\mathrm{TPH} 2^{+/+}$ (Sgk1: $+102 \% p<0.001 ;$ Gilz: $+130 \% p<0.001 ;$ Fkbp5: $+39 \%$ $p<0.01$ vs. $\mathrm{TPH} 2^{+/+} /$naïve) and $\mathrm{TPH} 2^{-/-}$rats $($Sgk1: $+117 \%$ $p<0.001$; Gilz: $+107 \% p<0.001$; Fkbp5: $+25 \% p<0.05$ vs. $\mathrm{TPH} 2^{-/-}$/naïve) (Figures 3D-F).

Also, P11 mRNA levels were affected by the stress $\left(F_{(1,35)}=6.158 p<0.05\right.$, two-way ANOVA). However, stress exposure induced a reduction in $P 11$ expression in both genotypes after stress exposure $\left(\mathrm{TPH}^{+/+}:-21 \% p>0.05\right.$ vs. $\mathrm{TPH} 2^{+/+} /$naïve; TPH $2^{-/-}:-28 \% p<0.05$ vs. TPH $2^{-/-} /$naïve). Moreover, TPH $2^{-/-}$rats showed increased levels of $P 11$ mRNA when compared to $\mathrm{TPH} 2^{+/+}\left(+43 \% p<0.05\right.$ vs. $\mathrm{TPH} 2^{+/+} /$naïve; Fisher's PLSD) (Figure 3G).

Finally, as shown in Figure $\mathbf{3 H}$, we did not find any changes in FoxO1 expression.

\section{Serotonin Deficiency Alters Clock Gene Expression Profile}

It has been demonstrated that acute stress exposure can influence the expression of Per1 thanks to the presence of a canonical GRE
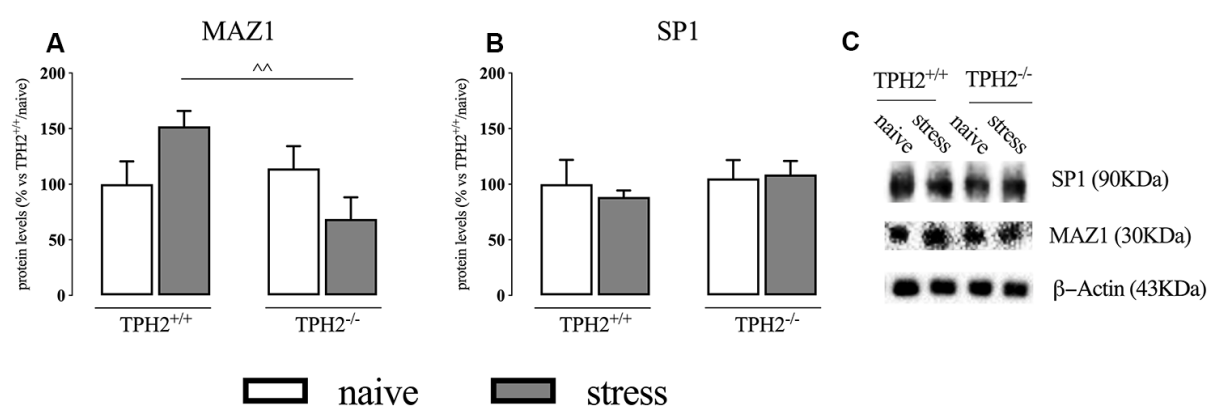

FIGURE 2 | Analysis of MAZ1 (A) and SP1 (B) protein levels in the whole homogenate of TPH2 $2^{+/+}$and TPH2 $2^{-/-}$stressed rats. (C) Representative western blot bands of MAZ1 and SP1. $\beta$-actin was used as an internal control. The data are expressed as a percentage of TPH $2^{+/+} /$naïve (set at $\left.100 \%\right)$ and are represented as mean \pm SEM of independent determinations. ${ }^{\wedge} p<0.01$ vs. TPH $2^{+/+} /$stress (two-way ANOVA with Fisher's PLSD). 

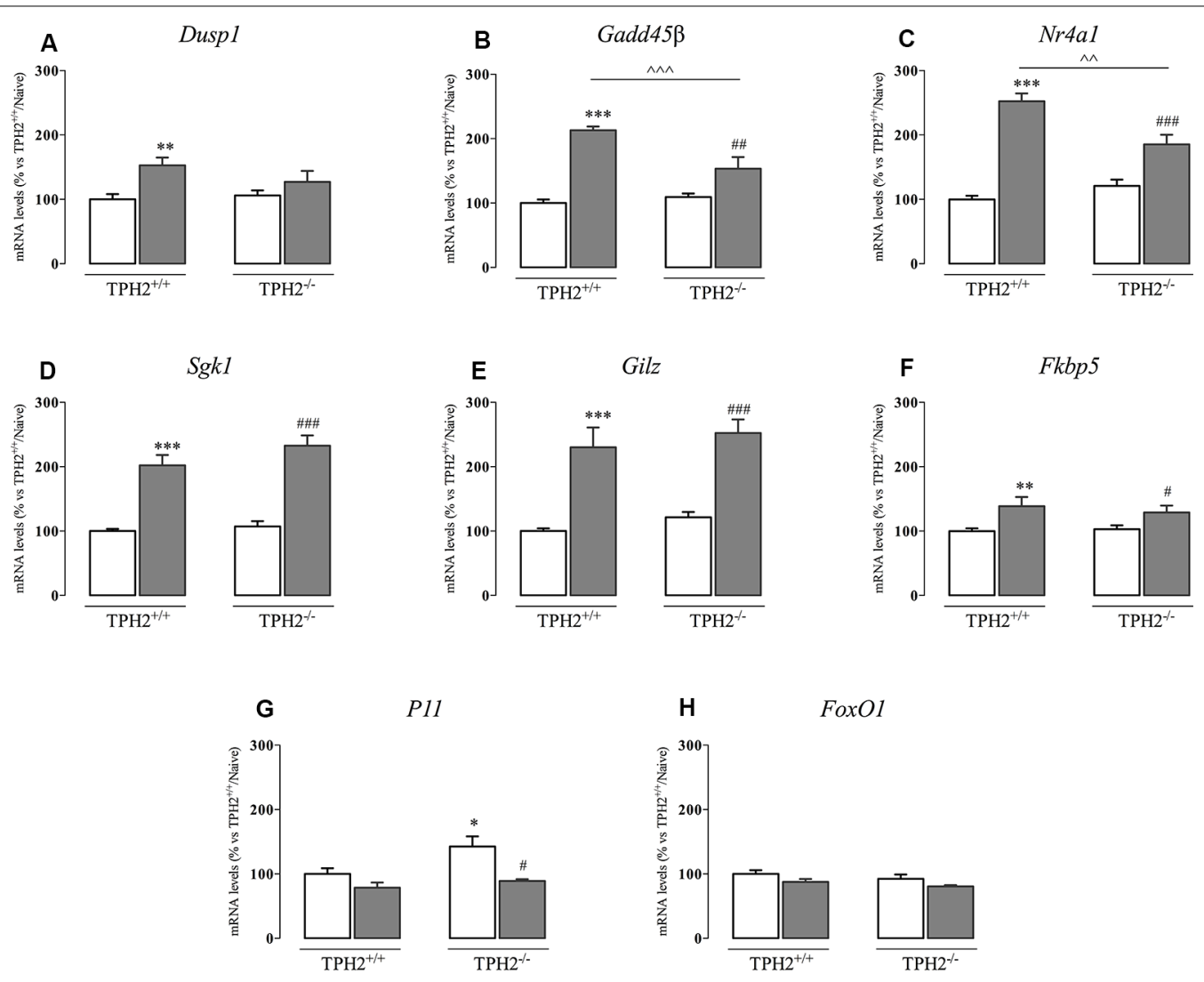

$\square$ naive

stress

FIGURE 3 | Analysis of glucocorticoid responsive gene expression in TPH $2^{+/+}$and TPH $2^{-/-}$stressed rats. The data are expressed as percentage of TPH $2^{+/+} /$naïve (set at 100\%) and are represented as mean \pm SEM of independent determinations. ${ }^{*} p<0.05 ;{ }^{* *} p<0.01 ;{ }^{* * *} p<0.001$ vs. TPH2 ${ }^{+/+} /$naïve; ${ }^{*} p<0.05$; ${ }^{* \#} p<0.01$; ${ }^{\# \# \#} p<0.001$ vs. TPH $2^{-/-} /$naïve; ${ }^{\wedge \wedge} p<0.01 ; \wedge \wedge p<0.001$ vs. TPH $2^{+/+} /$stress (two-way ANOVA with Fisher's PLSD).

(Yamamoto et al., 2005). Hence, seen the role of Per1 in the control of circadian rhythms, we measured the expression of the main components of the clock machinery.

In our animals, we found a significant genotype $\times$ stress interaction $\left(F_{(1,35)}=7.098 p<0.05\right.$, two-way ANOVA $)$ and a significant effect of the genotype $\left(F_{(1,35)}=15.130 p<0.01\right.$, two-way ANOVA) and of the stress $\left(F_{(1,35)}=115.534 p<0.001\right.$, two-way ANOVA) on Per1 expression. Indeed, as shown in Figure 4A, Per1 mRNA levels were significantly up-regulated in $\mathrm{TPH}_{2}^{+/+}$stressed rats in comparison to their naïve controls $\left(+80 \% p<0.001\right.$ vs. $\mathrm{TPH}^{+/+} /$naïve; Fisher's PLSD). This increase was present also in $\mathrm{TPH} 2^{-/-}$rats exposed to the restraint stress $\left(+52 \% p<0.001\right.$ vs. TPH $2^{-/-} /$naïve; Fisher's PLSD) even if the effect was more pronounced in $\mathrm{TPH}_{2}{ }^{/+}$ animals $\left(+28 \% p<0.01\right.$ vs. TPH $2^{+/+} /$stress; Fisher's PLSD).

Also, the expression of Per 2 showed a significant genotype $\times$ stress interaction $\left(F_{(1,34)}=5.725 p<0.05\right.$, two-way ANOVA) and a significant effect of the stress $\left(F_{(1,34)}=55.671 p<0.001\right.$, two-way ANOVA). However, Per2 expression was increased more in $\mathrm{TPH} 2^{-/-}$stressed animals than in $\mathrm{TPH} 2^{+/+}\left(\mathrm{TPH}^{+/+}\right.$: $+37 \% p<0.01$ vs. $\mathrm{TPH} 2^{+/+} /$stress; $\mathrm{TPH}^{-/-}:+75 \% p<0.001$; Fisher's PLSD). Indeed, the increased transcription of Per2 due to stress exposure was higher in $\mathrm{TPH} 2^{-/-}$compared to $\mathrm{TPH} 2^{+/+}$ animals $\left(+24 \% p<0.05\right.$ vs. $\mathrm{TPH} 2^{+/+} /$stress; Fisher's PLSD $)$ (Figure 4B).

We did not find any changes in Cry1 and Cry 2 expression (Figures 4C,D).

Similarly to what was observed in Perl expression, Reverb $\alpha$ mRNA levels were affected by the genotype and by the stress with a significant genotype $\times$ stress interaction (genotype: $F_{(1,35)}=10.544 p<0.05$; stress: $F_{(1,35)}=6.112$; genotype $\times$ stress interaction: $F_{(1,35)}=4.984 p<0.05 ; p<0.05$ two-way ANOVA). Specifically, Rev-erb $\alpha$ expression was increased in $\mathrm{TPH} 2^{+/+}$ stressed animals $\left(+31 \% p<0.01\right.$ vs. $\mathrm{TPH}^{+/+} /$naïve; Fisher's PLSD) while we did not observe any changes in $\mathrm{TPH} 2^{-/-}$rats (Figure 4E).

Finally, as shown in Figure 4F, Rev-erb $\beta$ expression showed a significant genotype $\times$ stress interaction $\left(F_{(1,34)}=4.789 p<0.05\right.$ two-way ANOVA). Indeed, we observed an up-regulation in Rev-erb $\beta$ expression in $\mathrm{TPH} 2^{-/-}$in comparison to $\mathrm{TPH}_{2}^{+/+}$ counterpart $\left(+20 \% \quad p<0.01\right.$ vs. $\mathrm{TPH}^{+/+} /$naïve; Fisher's PLSD). Moreover, Rev-erb $\beta$ levels were normalized in TPH $2^{-/-}$ rats after the stress $\left(-22 \% p<0.01\right.$ vs. $\mathrm{TPH} 2^{-/-} /$naïve; Fisher's PLSD). 

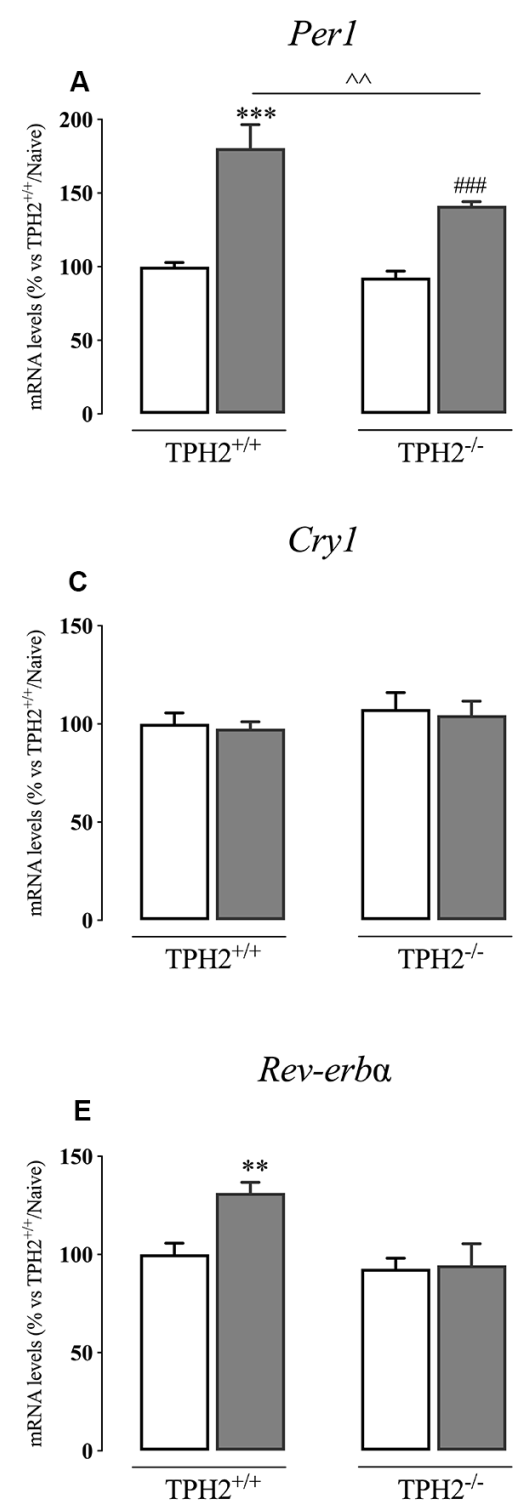
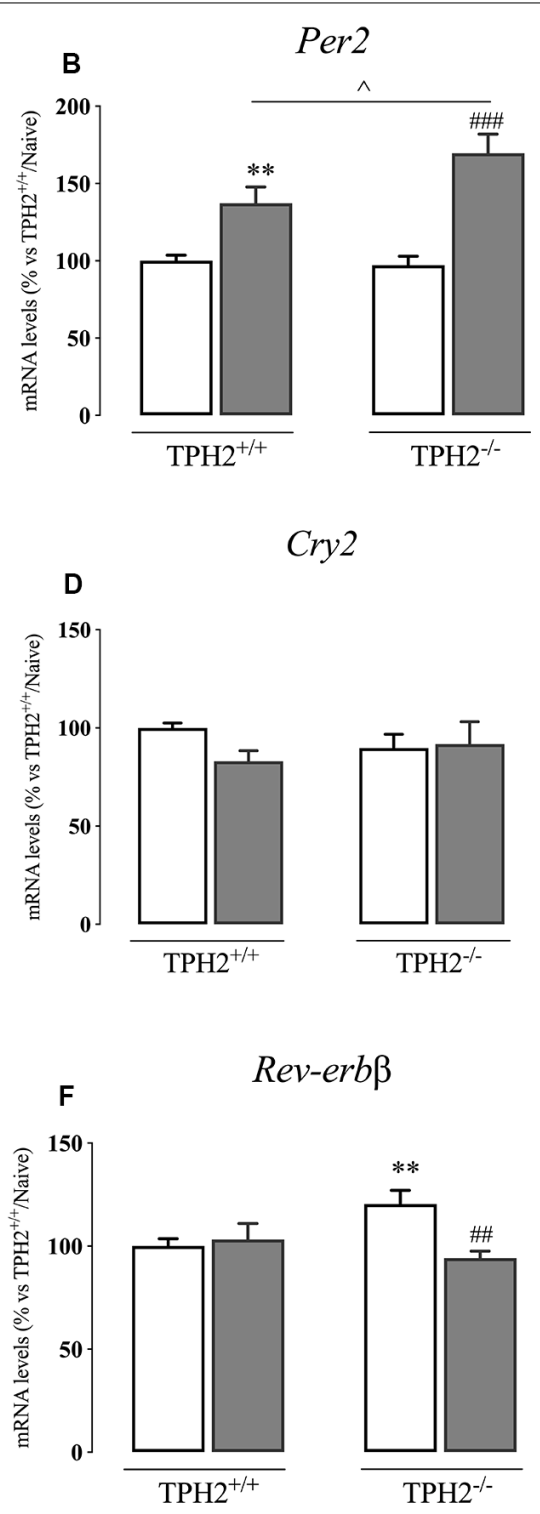

Stress

FIGURE 4 | Analysis of clock genes expression in TPH2 ${ }^{+/+}$and TPH2 $2^{-/-}$stressed rats. The data are expressed as percentage of TPH2 $2^{+/+} /$naïve (set at $\left.100 \%\right)$ and are represented as mean \pm SEM of independent determinations. ${ }^{* *} p<0.01 ;{ }^{* * *} p<0.001$ vs. TPH $2^{+/+} /$naïve; ${ }^{\# \#} p<0.01$; \#\#\# $p<0.001$ vs. TPH2 ${ }^{-/-} /$naïve; ${ }^{\wedge} p<0.05 ; \wedge \wedge<0.01$; vs. TPH $2^{+/+} /$stress (two-way ANOVA with Fisher's PLSD).

\section{DISCUSSION}

Our results show that serotonin deficiency interferes with the activation of the genomic pathway of GRs in response to an acute challenge. Moreover, it modulates the transcription of clock genes suggesting a possible alteration in circadian rhythms under stress challenge.

Interestingly, serotonin plays a crucial role in controlling brain functions not only at the basal level, but also in more dynamic situations. Indeed, we already demonstrated that the absence of serotonin in TPH2-deficient rats modulates neuroplastic mechanisms not only in basal conditions but also after acute stress exposure (Brivio et al., 2018).

The activation of the HPA axis is one of the first responses to a stressful experience. However, the downstream pathway that is activated after the release of corticosterone is very complex. In particular, once released from the adrenal glands, it targets the brain where it acts on its receptors widely localized in the cells. While membrane-bound GRs induce a rapid effect by modulating the release of neurotransmitters and mitochondrial 
activity through the so-called non-genomic pathway, cytosolic receptors are internalized into the nucleus where after the binding to GREs, they control the transcription of target genes (Schoneveld et al., 2004; Panettieri et al., 2019).

While in our previous study we found that the release of corticosterone following an acute challenge was independent of the serotonin levels in the brain (Brivio et al., 2018), here, we demonstrated that the internalization into the nucleus of the GRs induced by the exposure to an acute stressor happened specifically in $\mathrm{TPH} 2^{+/+}$, but not in $\mathrm{TPH} 2^{-/-}$rats. This suggests that the absence of serotonin in the brain does not influence the release of the hormone from the adrenal glands, but it interferes with the activation of the genomic pathway at the central level. In line, it has been demonstrated that the modulation of the serotonergic system by chronic treatment with antidepressant drugs enhances the translocation of GRs to the nuclear compartment (Molteni et al., 2009).

By investigating the levels of GR in the different subcellular compartments, we found that the lack of serotonin inhibits stress-induced translocation of GR to the nucleus. Since the direct influence of central serotonin on corticosterone release after acute stress is excluded by our previous experiments, another mechanism linking serotonin and GR functionality should exist. Most likely the cross-talk between these two systems appears at the level of interaction between downstream pathways of serotonin receptors with GR phosphorylation. Indeed, it has been demonstrated that the entry of GR to the nucleus is facilitated by the phosphorylation of specific serines (Wang et al., 2002) via activation of MAPKs and CDKs pathways (GalliherBeckley and Cidlowski, 2009). Since the activity of the MAPKs is modulated by 5-HT2A and 5HT1A receptors (Banes et al., 1999; Errico et al., 2001), blunted activation of 5-HT receptor signaling in the absence of ligand may be responsible for the lack of GR phosphorylation and its subsequent translocation to the nucleus upon acute stress. Furthermore, it has been shown that GR phosphorylation is also controlled by BDNF signaling (Arango-Lievano et al., 2015). We previously demonstrated that $\mathrm{TPH} 2^{-/-}$rats showed a reduced $B d n f$ expression after the exposure to $1 \mathrm{~h}$ of acute restraint stress compared to the wild type counterpart (Brivio et al., 2018). This may lead to a decreased ability of the neurotrophin-related pathways to phosphorylate GRs.

Interestingly, the GREs are characterized by the presence of GC enriched binding sites that are targeted by transcription factors such as MAZ1 and SP1. These transcription factors in the proximity to GREs can potentiate the GR binding and therefore, enhance the transcriptional activation of target genes (Datson et al., 2011). Here, in line with the increase of GR in the nucleus, we observed increased levels of MAZ1 in $\mathrm{TPH} 2^{+/+}$ stressed animals contrasting with a slight decrease in $\mathrm{TPH} 2^{-/-}$ stressed animals. This data suggests that the reduced activation of the genomic pathway in $\mathrm{TPH} 2^{-/-}$rats in response to the acute challenge may be caused not only by the lack of GRs translocation per se but also by the reduced levels of this cofactor. On the contrary, SP1 was not altered after stress exposure. Hence, these two cofactors may influence the interaction between GRs and their responsive elements with different timing.
Furthermore, even if both MAZ1 and SP1 can interact with GRs, SP1 seems to be mainly related to mineralocorticoid receptors (Meinel et al., 2013) suggesting its potential implication in physiological conditions and not in response to an acute challenge.

The GR, as a nuclear receptor, acts as a transcriptional regulator interfering with the transcription of genes carrying the GRE in their regulatory sequence (Gray et al., 2017). In line, we recently demonstrated that the translocation into the nucleus is paralleled by an up-regulation of the expression of genes containing the GRE such as Gadd45 $\beta$, Sgk1, Nr4a1, and Dusp1 (Calabrese et al., 2020). Here, we observed that GR responsive genes were activated more in $\mathrm{TPH}^{+/+}$than in $\mathrm{TPH} 2^{-/-}$rats. In particular, Dusp 1 mRNA was increased specifically in $\mathrm{TPH} 2^{+/+}$while no changes were observed in $\mathrm{TPH}^{-/-}$rats. Similarly, Gadd45 $\beta$ and $S g k 1$ mRNAs were increased in both genotypes, but more in $\mathrm{TPH}^{+/+}$than in $\mathrm{TPH} 2^{-/-}$rats. This suggests the ability of serotonin to interfere with their transcription. Indeed, several studies showed that pharmacological or genetic alterations of the serotonergic system can modulate their expression (Gonzalez-Nicolini and McGinty, 2002; Duric et al., 2010; Calabrese et al., 2013). However, other glucocorticoid responsive genes were equally upregulated after the acute challenge in the two genotypes. This could be due to other factors that can influence their expression and that may contribute to the stress response (Lang et al., 2014; Zannas et al., 2016). Furthermore, it could not be excluded that the absence of serotonin may modify the epigenetic make-up of some GR related genes via the histone-serotonylation mechanism (Farrelly et al., 2019), and therefore their transcription after an acute challenge.

As mentioned, serotonin is involved in the control of various physiological functions and, among them, the circadian system is braided with the neurotransmitter both anatomically and genetically (Deurveilher and Semba, 2005). Furthermore, it has been demonstrated that genes involved in the clock gene machinery can be affected by acute stress exposure (Yamamoto et al., 2005). Interestingly, Per1 expression showed a similar pattern as Gadd45 $\beta$ and $N r 4 a 1$, with increased transcription in $\mathrm{TPH} 2^{+/+}$compared to $\mathrm{TPH} 2^{-/-}$rats exposed to stress. On the contrary, Per 2 mRNA levels were increased more in $\mathrm{TPH} 2^{-/-}$than in $\mathrm{TPH} 2^{+/+}$rats. This different pattern could be due to a different responsiveness of Per1 and Per2 to GRs. Indeed, while Per 1 carries a canonical GRE, Per2 has an intronic binding sequence that confers GR responsiveness to the gene (So et al., 2009). Moreover, Rev-erb $\alpha$ expression was up-regulated by stress in $\mathrm{TPH} 2^{+/+}$but not in $\mathrm{TPH} 2^{-/-}$rats while $\operatorname{Rev}$-erb $\beta$ expression showed a basal increase in $\mathrm{TPH} 2^{-/-}$rats while a normalization occurred after the acute stress. Since Rev-erbs are responsible for the proper interlocking feedback loop in the clock machinery (Lowrey and Takahashi, 2011), the modulations observed could indicate alterations in the circadian rhythm at basal level and in the feedback to clock gene activation after the stress.

All in all, our data confirm the involvement of serotonin in the proper stress response and that the absence of the neurotransmitter in the brain interferes with the functionality 
of the HPA axis in the PFC modulating the genomic pathway activation. Similarly, serotonin depletion affected clock gene activity at basal level and in response to the acute challenge, suggesting a potential link between serotonin, stress and circadian rhythms.

\section{DATA AVAILABILITY STATEMENT}

The datasets generated for this study are available on request to the corresponding author.

\section{ETHICS STATEMENT}

The animal study was reviewed and approved by LAGeSo, Berlin, Germany.

\section{AUTHOR CONTRIBUTIONS}

FC and NA: conception and study design. GS, PB, PP, and MT: performed experiments. GS, PB, FC, and NA: performed data analysis and interpretation of the data. GS: drafted the manuscript. FC, NA, and MB critically revised the manuscript.

\section{REFERENCES}

Adzic, M., Djordjevic, J., Djordjevic, A., Niciforovic, A., Demonacos, C., Radojcic, M., et al. (2009). Acute or chronic stress induce cell compartmentspecific phosphorylation of glucocorticoid receptor and alter its transcriptional activity in Wistar rat brain. J. Endocrinol. 202, 87-97. doi: 10.1677/joe08-0509

Akiskal, H. S., and McKinney, W. T. (1973). Psychiatry and pseudopsychiatry. Arch. Gen. Psychiatry 28, 367-373. doi: 10.1001/archpsyc.1973. 01750330055010

Akiskal, H. S., and McKinney, W. T. Jr. (1975). Overview of recent research in depression: integration of ten conceptual models into a comprehensive clinical frame. Arch. Gen. Psychiatry 32, 285-305. doi: 10.1001/archpsyc.1975. 01760210019001

Arango-Lievano, M., Lambert, W. M., Bath, K. G., Garabedian, M. J., Chao, M. V., and Jeanneteau, F. (2015). Neurotrophic-priming of glucocorticoid receptor signaling is essential for neuronal plasticity to stress and antidepressant treatment. Proc. Natl. Acad. Sci. U S A 112, 15737-15742. doi: 10.1073/pnas. 1509045112

Banes, A., Florian, J. A., and Watts, S. W. (1999). Mechanisms of 5hydroxytryptamine $(2 \mathrm{~A})$ receptor activation of the mitogen-activated protein kinase pathway in vascular smooth muscle. J. Pharmacol. Exp. Ther. 291, 1179-1187.

Breslau, N., and Davis, G. C. (1986). Chronic stress and major depression. Arch. Gen. Psychiatry 43, 309-314. doi: 10.1001/archpsyc.1986.01800040015003

Brivio, P., Corsini, G., Riva, M. A., and Calabrese, F. (2019). Chronic vortioxetine treatment improves the responsiveness to an acute stress acting through the ventral hippocampus in a glucocorticoid-dependent way. Pharmacol. Res. 142, 14-21. doi: 10.1016/j.phrs.2019.02.006

Brivio, P., Sbrini, G., Peeva, P., Todiras, M., Bader, M., Alenina, N., et al. (2018). TPH2 deficiency influences neuroplastic mechanisms and alters the response to an acute stress in a sex specific manner. Front. Mol. Neurosci. 11:389. doi: 10.3389/fnmol.2018.00389

Brivio, P., Sbrini, G., Riva, M. A., and Calabrese, F. (2020). Acute stress induces cognitive improvement in the novel object recognition task by transiently modulating bdnf in the prefrontal cortex of male rats. Cell. Mol. Neurobiol. doi: 10.1007/s10571-020-00793-7 [Epub ahead of print].

Calabrese, F., Brivio, P., Sbrini, G., Gruca, P., Lason, M., Litwa, E., et al. (2020). Effect of lurasidone treatment on chronic mild stress-induced behavioural
All authors critically reviewed the content and approved the final version for publication.

\section{FUNDING}

This work was supported by grants from MIUR ProgettoEccellenza to FC; by PRIN2017 to FC, by piano sostegno Alla ricerca UNIMI, linea 2 azione A to FC, by the DAAD-MIUR Joint Mobility Program to NA and FC; by German Federal Ministry of Education and Research under the framework of the EU-ERA-NET NEURON project RESPOND to MB and NA; by the project 51143531 of the St. Petersburg State University and the Volkswagen Foundation to NA. GS was supported by cycle XXXIV of the doctorate in Experimental and Clinical Pharmacological Sciences, Department of Pharmacological and Biomolecular Sciences, Università' Degli Studi di Milano.

\section{ACKNOWLEDGMENTS}

We thank Susanne da Costa Goncalves, Annegret Dahlke, Laura Rosenzweig, and Monique Bergemann for the excellent technical assistance.

deficits in male rats: the potential role for glucocorticoid receptor signalling J. Psychopharmacol. 34, 420-428. doi: 10.1177/0269881119895547

Calabrese, F., Guidotti, G., Middelman, A., Racagni, G., Homberg, J., and Riva, M. A. (2013). Lack of serotonin transporter alters BDNF expression in the rat brain during early postnatal development. Mol. Neurobiol. 48, 244-256. doi: $10.1007 / \mathrm{s} 12035-013-8449-\mathrm{Z}$

Caspi, A., Sugden, K., Moffitt, T. E., Taylor, A., Craig, I. W., Harrington, H. L., et al. (2003). Influence of life stress on depression: moderation by a polymorphism in the 5-HTT gene. Science 301, 386-389. doi: 10.1126/science.1083968

Datson, N. A., Polman, J. A. E., de Jonge, R. T., van Boheemen, P. T. M., van Maanen, E. M. T., Welten, J., et al. (2011). Specific regulatory motifs predict glucocorticoid responsiveness of hippocampal gene expression. Endocrinology 152, 3749-3757. doi: 10.1210/en.2011-0287

Deurveilher, S., and Semba, K. (2005). Indirect projections from the suprachiasmatic nucleus to major arousal-promoting cell groups in rat: implications for the circadian control of behavioural state. Neuroscience 130, 165-183. doi: 10.1016/j.neuroscience.2004.08.030

Duman, R. S., and Monteggia, L. M. (2006). A neurotrophic model for stressrelated mood disorders. Biol. Psychiatry 59, 1116-1127. doi: 10.1016/j.biopsych. 2006.02.013

Duric, V., Banasr, M., Licznerski, P., Schmidt, H. D., Stockmeier, C. A., Simen, A. A., et al. (2010). A negative regulator of MAP kinase causes depressive behavior. Nat. Med. 16, 1328-1332. doi: 10.1038/nm.2219

Errico, M., Crozier, R. A., Plummer, M. R., and Cowen, D. S. (2001). 5-HT7 receptors activate the mitogen activated protein kinase extracellular signal related kinase in cultured rat hippocampal neurons. Neuroscience 102, 361-367. doi: 10.1016/s0306-4522(00)00460-7

Farrelly, L. A., Thompson, R. E., Zhao, S., Lepack, A. E., Lyu, Y., Bhanu, N. V., et al. (2019). Histone serotonylation is a permissive modification that enhances TFIID binding to H3K4me3. Nature 567, 535-539. doi: 10.1038/s41586-0191024-7

Fitzpatrick, P. F. (1999). Tetrahydropterin-dependent amino acid hydroxylases. Annu. Rev. Biochem. 68, 355-381. doi: 10.1146/annurev.biochem.68.1.355

Galliher-Beckley, A. J., and Cidlowski, J. A. (2009). Emerging roles of glucocorticoid receptor phosphorylation in modulating glucocorticoid hormone action in health and disease. IUBMB Life 61, 979-986. doi: 10.1002/ iub. 245

Gonzalez-Nicolini, V., and McGinty, J. F. (2002). Gene expression profile from the striatum of amphetamine-treated rats: a cDNA array and in situ 
hybridization histochemical study. Brain Res. Gene Expr. Patterns 1, 193-198. doi: $10.1016 /$ s1567-133x(02)00017-0

Gray, J. D., Kogan, J. F., Marrocco, J., and McEwen, B. S. (2017). Genomic and epigenomic mechanisms of glucocorticoids in the brain. Nat. Rev. Endocrinol. 13, 661-673. doi: 10.1038/nrendo.2017.97

Herman, J. P., Figueiredo, H., Mueller, N. K., Ulrich-Lai, Y., Ostrander, M. M., Choi, D. C., et al. (2003). Central mechanisms of stress integration: hierarchical circuitry controlling hypothalamo-pituitary-adrenocortical responsiveness. Front. Neuroendocrinol. 24, 151-180. doi: 10.1016/j.yfrne.2003. 07.001

Kaplan, K., Echert, A. E., Massat, B., Puissant, M. M., Palygin, O., Geurts, A. M., et al. (2016). Chronic central serotonin depletion attenuates ventilation and body temperature in young but not adult Tph2 knockout rats. J. Appl. Physiol. 120, 1070-1081. doi: 10.1152/japplphysiol.01015.2015

Lang, F., Stournaras, C., and Alesutan, I. (2014). Regulation of transport across cell membranes by the serum-and glucocorticoid-inducible kinase SGK1. Mol. Membr. Biol. 31, 29-36. doi: 10.3109/09687688.2013.874598

Lowrey, P. L., and Takahashi, J. S. (2011). Genetics of circadian rhythms in mammalian model organisms. Adv. Genet. 74, 175-230. doi: 10.1016/b978-012-387690-4.00006-4

Lupien, S. J., Juster, R. P., Raymond, C., and Marin, M. F. (2018). The effects of chronic stress on the human brain: from neurotoxicity, to vulnerability, to opportunity. Front. Neuroendocrinol. 49, 91-105. doi: 10.1016/j.yfrne.2018. 02.001

McEwen, B. S. (2007). Physiology and neurobiology of stress and adaptation: central role of the brain. Physiol. Rev. 87, 873-904. doi: 10.1152/physrev.00041. 2006

Meinel, S., Ruhs, S., Schumann, K., Strätz, N., Trenkmann, K., Schreier, B., et al. (2013). Mineralocorticoid receptor interaction with SP1 generates a new response element for pathophysiologically relevant gene expression. Nucleic Acids Res. 41, 8045-8060. doi: 10.1093/nar/gkt581

Molteni, R., Calabrese, F., Cattaneo, A., Mancini, M., Gennarelli, M., Racagni, G., et al. (2009). Acute stress responsiveness of the neurotrophin bdnf in the rat hippocampus is modulated by chronic treatment with the antidepressant duloxetine. Neuropsychopharmacology 34, 1523-1532. doi: 10.1038/npp. 2008.208

Mosienko, V., Beis, D., Pasqualetti, M., Waider, J., Matthes, S., Qadri, F., et al. (2015). Life without brain serotonin: reevaluation of serotonin function with mice deficient in brain serotonin synthesis. Behav. Brain Res. 277, 78-88. doi: 10.1016/j.bbr.2014.06.005

Nader, N., Chrousos, G. P., and Kino, T. (2010). Interactions of the circadian CLOCK system and the HPA axis. Trends Endocrinol. Metab. 21, 277-286. doi: 10.1016/j.tem.2009.12.011

Ottenhof, K. W., Sild, M., Lévesque, M. L., Ruhé, H. G., and Booij, L. (2018). TPH2 polymorphisms across the spectrum of psychiatric morbidity: a systematic review and meta-analysis. Neurosci. Biobehav. Rev. 92, 29-42. doi: 10.1016/j.neubiorev.2018.05.018

Pan, L., and Gilbert, F. (1992). Activation of $5-\mathrm{HT}_{1 A}$ receptor subtype in the paraventricular nuclei of the hypothalamus induces $\mathrm{CRH}$ and ACTH release in the rat. Neuroendocrinology 56, 797-802. doi: 10.1159/000126332
Panettieri, R. A., Schaafsma, D., Amrani, Y., Koziol-White, C., Ostrom, R., and Tliba, O. (2019). Non-genomic effects of glucocorticoids: an updated view. Trends Pharmacol. Sci. 40, 38-49. doi: 10.1016/j.tips.2018.11.002

Paxinos, G., and Watson, C. (2007). The Rat Brain in Stereotaxic Coordinates Sixth Edition. San Diego: Elsevier Academic Press.

Revollo, J. R., and Cidlowski, J. A. (2009). Mechanisms generating diversity in glucocorticoid receptor signaling. Ann. N Y Acad. Sci. 1179, 167-178. doi: 10.1111/j.1749-6632.2009.04986.x

Schoneveld, O. J. L. M., Gaemers, I. C., and Lamers, W. H. (2004). Mechanisms of glucocorticoid signalling. Biochim. Biophys. Acta 1680, 114-128. doi: 10.1016/j. bbaexp.2004.09.004

So, A. Y. L., Bernal, T. U., Pillsbury, M. L., Yamamoto, K. R., and Feldman, B. J. (2009). Glucocorticoid regulation of the circadian clock modulates glucose homeostasis. Proc. Natl. Acad. Sci. U S A 106, 17582-17587. doi: 10.1073/pnas. 0909733106

Stuss, D. T., and Knight, R. T. (2009). Principles of Frontal Lobe Function. New York, NY: Oxford University Press.

Trapp, T., Rupprecht, R., Castrén, M., Reul, J. M. H. M., and Holsboer, F. (1994). Heterodimerization between mineralocorticoid and glucocorticoid receptor: a new principle of glucocorticoid action in the CNS. Neuron 13, 1457-1462. doi: 10.1016/0896-6273(94)90431-6

Van de Kar, L. D., Javed, A., Zhang, Y., Serres, F., Raap, D. K., and Gray, T. S. (2001). 5-HT2A receptors stimulate ACTH, corticosterone, oxytocin, renin, and prolactin release and activate hypothalamic CRF and oxytocin-expressing cells. J. Neurosci. 21, 3572-3579. doi: 10.1523/JNEUROSCI.21-10-03572.2001

Walther, D. J., Peter, J. U., Bashammakh, S., Hörtnagl, H., Voits, M., Fink, H., et al. (2003). Synthesis of serotonin by a second tryptophan hydroxylase isoform. Science 299:76. doi: 10.1126/science.1078197

Wang, Z., Frederick, J., and Garabedian, M. J. (2002). Deciphering the phosphorylation "code" of the glucocorticoid receptor in vivo. J. Biol. Chem. 277, 26573-26580. doi: 10.1074/jbc.M110530200

Yamamoto, T., Nakahata, Y., Tanaka, M., Yoshida, M., Soma, H., Shinohara, K., et al. (2005). Acute physical stress elevates mouse Period1 mRNA expression in mouse peripheral tissues via a glucocorticoid-responsive element. J. Biol. Chem. 280, 42036-42043. doi: 10.1074/jbc.m509600200

Zannas, A. S., Wiechmann, T., Gassen, N. C., and Binder, E. B. (2016). Genestress-epigenetic regulation of FKBP5: clinical and translational implications. Neuropsychopharmacology 41, 261-274. doi: 10.1038/npp.2015.235

Conflict of Interest: The authors declare that the research was conducted in the absence of any commercial or financial relationships that could be construed as a potential conflict of interest.

Copyright (๑) 2020 Sbrini, Brivio, Peeva, Todiras, Bader, Alenina and Calabrese. This is an open-access article distributed under the terms of the Creative Commons Attribution License (CC BY). The use, distribution or reproduction in other forums is permitted, provided the original author(s) and the copyright owner(s) are credited and that the original publication in this journal is cited, in accordance with accepted academic practice. No use, distribution or reproduction is permitted which does not comply with these terms. 